\title{
Rehabilitation and recovery for ethnic minority patients with severe mental illness ${ }^{\dagger}$
}

\section{Martin Rotenberg}

\begin{abstract}
SUMMARY
There is growing evidence to support recovery and rehabilitation services and interventions for people with severe mental illness (SMI). However, those from ethnic minority communities face inequitable outcomes and access to mental health services and poorer functional outcomes. This article reviews the evidence and discusses facilitators and barriers in the recovery journey of people with SMI from ethnic minority groups. Although there is limited evidence for specific interventions for ethnic minority patients, areas for future study and action are discussed.
\end{abstract}

\section{LEARNING OBJECTIVES}

After reading this article you will be able to:

- understand the scope of rehabilitation practices and interventions and evidence for use with ethnic minority patients with severe mental illness

- describe differences and similarities in the conceptualisation of recovery by majority and minority ethnic communities

- appreciate facilitators and barriers to rehabilitation and recovery for ethnic minority patients with SMI.

\section{DECLARATION OF INTEREST}

None.

\section{KEYWORDS}

Rehabilitation; psychosocial interventions; service users; transcultural psychiatry; stigma and discrimination.

Equitable outcomes with respect to mental health services are important from both the policy and care perspectives. Poor functional outcomes for individuals with severe mental illness (SMI) - schizophrenia and schizoaffective disorder in particular have been a challenge and contribute to greater disability and burden of illness. There is a wide range of psychosocial rehabilitation practices and interventions that can be provided to people with SMI (Box 1), and evidence-based interventions such as
BOX 1 Scope of rehabilitation practices and interventions

- Recovery and rehabilitation assessment

- Illness and comorbidity (self-Imanagement

- Cognitive impairments and remediation

- Care management

- Housing

- Employment and education

- Social functioning

- Family interventions

- Criminal justice and risk/safety

- Stigma and cultural competence

- Self-help and peer support

(Adapted from Rotenberg 2017a)

supported employment and supported housing, as well as promising practices such as supported education, are increasingly studied. However, questions surrounding access, acceptability and outcomes for patients from ethnic minority groups and communities has not been a major focus of study.

Over two decades ago the $Æ$ ESOP study was set up to follow a cohort of more than 500 ethnoculturally diverse patients with a first episode of psychosis. Ten-year follow-up findings on clinical and social outcomes as well as service use were published in 2015 (Revier 2015). With FSOP's recent publication of functional outcomes for White majority and Black minority patients (Morgan 2017) it is perhaps time for a critical evaluation of psychosocial rehabilitation services provided to people with SMI, particularly those from ethnic minority backgrounds, who often face additional disparities and disadvantage in their recovery journey (Department of Health 2003).

Specific attention should be given to rehabilitation for ethnic minority patients, as they face more complicated pathways to care and increased rates of involuntary detention and coercion in care (Department of Health 2003; Morgan 2004), all of
Martin Rotenberg, MD, is a psychiatry resident at the University of Toronto, Canada. He has a clinical interest in psychiatric rehabilitation and his research focuses on socioenvironmental factors that impact care in schizophrenia spectrum disorders.

Correspondence Dr Martin

Rotenberg, Centre for Addiction and Mental Health, 33 Russell Street, Room 2017, Toronto, Ontario M5S 3B1, Canada. Email: marty.rotenberg@mail.utoronto.ca

First received 9 Aug 2018

Final revision 26 Nov 2018 Accepted 6 Mar 2019

\section{Copyright and usage}

(C) The Royal College of Psychiatrists 2019

${ }^{\dagger} \mathrm{A}$ version of this article was presented at the annual meeting of the Royal College of Psychiatrists' Faculty of Rehabilitation and Social Psychiatry in Bournemouth, November 2017, and received the Douglas Bennett Prize. 
which may complicate recovery-oriented care. Furthermore, the incidence of primary psychotic illnesses may be elevated in some ethnic minority communities (Anderson 2015), which may influence levels of need and have implications for service planning. In addition, ethnic minority patients are often more marginalised at baseline from a social, educational and financial perspective prior to engaging with services (Nagendra 2017).

As noted above, data from the 10-year follow-up of the ESOP cohort (Morgan 2017) suggest that functional outcomes - specifically employment outcomes - for BME individuals with psychosis are even poorer than those for White British individuals (which are also very poor). The Black Caribbean participants were approximately 5 times more likely than the White British to have been employed for less than $75 \%$ of the follow-up period. Only $6 \%$ $(n=3)$ of the Black Caribbean participants and only $5 \%(n=1)$ of the Black African participants who were unemployed at baseline were employed at the end of the follow-up period. Black African and Black Caribbean participants also scored lower on a measure of social functioning than the White British participants. These findings add to the growing evidence of disparities in functional outcomes among ethnic minority patients (Birchwood 1992; Agius 2008; Eack 2012) - specifically, lower likelihood of return to work or higher education, poorer assessment of global functioning and higher rates of relapse and readmission. This is particularly concerning given increasing evidence of the success of psychiatric rehabilitation practices for majority communities (Rotenberg 2017a) and calls to action to reduce disparities.

The overall goal of this article is to review evidence for psychosocial rehabilitation and recoveryoriented practices and interventions for ethnic minority patients with SMI, draw further attention to problems regarding outcomes and equity, and consider action than might improve care and outcomes in all peoples with SMI. Purely pharmacological and psychological interventions are, of course, important in providing comprehensive psychiatric care, but I do not discuss them here (however, note disparities across patients exist with these evidence-based modalities). Some of the terms used in this article are defined in Box 2, and Box 3 defines rehabilitation and recovery.

\section{Recovery as a concept}

Recovery in mental healthcare can be conceptualised as a set of processes and/or outcomes that support a personally meaningful life despite and beyond having a mental illness (Rotenberg 2017a). Ethnic minority communities may have different
BOX 2 Terminology

Severe mental illness (SMI) - diagnoses that typically involve psychosis or require high levels of care, commonly schizophrenia, schizoaffective disorder and bipolar disorder.

Service user, client and patient - terms used to refer to individuals who utilise mental health services. The word chosen may vary depending on the writer's clinical discipline, the orientation of care and context.

Black and minority ethnic (BME) groups - a term used in the UK to refer to individuals from Black, Asian, mixed or other ethnic groups, to differentiate from the White majority; the acronym BAME (Black, Asian and minority ethnic) is also often used.

Ethnic minority - general term used in this article to differentiate from the White majority (effort has been made to be specific in describing the ethnicity of participants of the studies mentioned).

conceptualisations of mental illness (Cinnirella 1999) and different patterns and levels of stigma (Knifton 2010). On the basis of these differences it has previously been hypothesised that ethnic minority communities may also have different perceptions of recovery. However, recent research suggests that both majority and minority ethnic communities may share a common view of recovery, with the exception that ethnic minority groups may see a greater role for spirituality and religion in recovery (Shank 2015; Whitley 2016). These findings suggest that overall, patients from minority ethnic groups should benefit equally from rehabilitation services - which are primarily oriented within a recovery framework - if they are able to access and utilise them.

\section{Synthesis of evidence: recovery and rehabilitation services in action}

\section{Vocational rehabilitation}

Employment is one of the more widely studied domains of rehabilitation and is viewed as an important functional marker. A 2016 Cochrane

BOX 3 Rehabilitation and recovery defined

Psychosocial rehabilitation - also referred to as psychiatric rehabilitation - is an approach that promotes recovery, community integration and improving the quality of life of individuals with a mental illness via services that increase capacity for individuals to be satisfied and successful in the environment of their choosing.

Recovery - a set of processes and/or outcomes that support a personally meaningful life despite and beyond having a mental illness. 
review (Kinoshita 2013) found interventions such as supported employment and, specifically, the individual placement and support (IPS) model, to be effective in improving vocational outcomes in individuals with SMI. A randomised controlled trial comparing supported employment with other vocational interventions conducted in the USA found rates of competitive employment among Latino and African American participants with SMI randomised to supported employment similar to those among White participants receiving the same support (Mueser 2014). However, in practice in the USA, outside the confines of such trials, ethnic minority patients are often offered different services and face challenges accessing vocational rehabilitation services (Olney 2002). Lukyanova et al (2014), for example, found that ethnic minority individuals with mental illness engaging in vocational rehabilitation have poorer employment outcomes than White individuals.

\section{Care and service delivery models}

Intensive case management (Dieterich 2017) and assertive community treatment (Bond 2001) are both service delivery models that can operate within a recovery framework and may reduce hospital admissions and improve housing and social stability. In the UK, assertive outreach teams provide modified versions of assertive community treatment. Many of these teams were disbanded following a study (dubbed REACT) that found community mental health teams to perform equally as well as assertive community treatment teams in reducing inpatient bed utilisation and on social and clinical measures, even though patients were found to be more satisfied and engaged with assertive outreach services (Killaspy 2006).

Ethnic matching has been studied in several trials. In the UK, Black patients randomised to an ethnically matched assertive outreach team or to treatment as usual reported high satisfaction ratings with the ethnically matched assertive outreach; however, there was no reduction in hospital admissions despite a reduction in symptoms (Bhugra 2011). In Canada, observational data showed that an ethnoculturally specific assertive community treatment team that matched patients, primarily from East Asian communities, to primary workers on the basis of language and ethnicity was successful in reducing hospital admissions and was highly acceptable to patients and family members (Yang 2005; Chow 2009). Ethnic matching in service and care delivery may have a role in increasing access to additional rehabilitation services (Sass 2009) and improving engagement and satisfaction with services, particularly if mistrust has developed because of previous contacts.
Despite the satisfaction and acceptability of matching in these studies, it should also be noted that it may not be a patient's preference to work with providers from the same or a similar community, particularly if the community is small, because of concerns surrounding confidentiality.

\section{Supported housing}

Supported housing - the provision of independent housing with mental health support - can improve housing outcomes (Rosenheck 2003). In Canada, patients from ethnic minority groups who were homeless or precariously housed were randomised to immediate housing with intensive case management or to 'treatment as usual'. Those in the immediate housing group showed significant improvements in housing stability and community functioning and fewer hospital admissions compared with those who received treatment as usual (Stergiopoulos 2015).

Although supported housing shows promise in improving recovery, ethnic minority patients may face greater problems keeping their housing placements once obtained. For example, for women from ethnic minority groups, gender itself may be a barrier to maintaining stable housing, as many face additional marginalisation from the health and social service sectors and also from their own ethnic communities (Kidd 2013). There is also little evidence on the uptake of housing services by ethnic minority patients and how acceptable they find such services.

\section{Cultural adaptation and responsiveness of interventions and practices}

There is increasing interest in adapting psychotherapeutic and psychosocial interventions for particular ethnocultural groups (Bernal 2009). There has been debate about how transferable interventions may be, considering differences in ideas and values regarding mental illness. However, values regarding recovery may be more similar between ethnocultural groups, and recovery interventions and practices therefore have the potential to be more easily adapted despite linguistic, cultural and social differences.

There are limited examples of rehabilitation services that have been formally adapted for specific minority communities or for ethnically diverse communities. There is ongoing work on increasing the cultural responsiveness of the recovery and rehabilitation assessment process (Yamada 2008) and of peer support for patients with psychosis from Latino and African American communities (Tondora 2010), but implementation of these projects has yet to be studied or reported.

It may be important to further examine whether there is a role and need to adapt other rehabilitation 
practices and interventions that have growing evidence bases (e.g. supported employment) and evaluate outcomes and what wider lessons may be learned.

\section{Barriers and facilitators}

There are important barriers and facilitators to rehabilitation and recovery for ethnic minority patients (Box 4). A significant barrier may be the fact ethnic minority patients experience more frequent involuntary and coercive hospital admissions. Inpatient admissions may not be supportive of rehabilitation and recovery (Whitley 2016), and the use of coercion runs against fundamental ethical principles of rehabilitation and the recovery model, which values the upholding of self-determination and autonomy (Rotenberg 2011, 2017a). In general, financial stress and stigma have been identified as major barriers to recovery across all patients (Whitley 2016). Major psychosocial stressors may be experienced differently by patients from different ethnocultural groups (Rotenberg 2017b) and at baseline, minority communities may be more marginalised. This may be a function of racism, which can operate at individual, communal and societal levels, and can erode service quality and undermine social determinants of health. Furthermore, basic accessibility problems, which include the language of service provision and location of services, are also important logistical factors that act as barriers.

Positive drivers that facilitate rehabilitation for patients from ethnic minority groups include building on the increasing interest in adapting and implementing practices and services in a culturally responsive manner (Yamada 2008; Tondora 2010). It is also possible to increase trust in mental

BOX 4 Barriers and facilitators to rehabilitation and recovery for ethnic minority patients

Barriers

- Coercion

- Pathways to care

- Logistics (language, location, finances)

- Marginalisation

- Racism

Facilitators

- Interest among service providers

- Service providers' education and competency

- Trust

- Ethno-specific services/matching

- Alternative care approaches (e.g. spiritual and religious community involvement) healthcare and rehabilitation via positive interactions with care providers, particularly those from minority backgrounds (Ali 2017), which can be facilitated by ethno-specific matching (Yang 2005; Chow 2009; Bhugra 2011). It may also be important to engage religious and spiritual communities that patients affiliate with, as these may play an important role in recovery (Whitley 2016) and may facilitate access to additional resources and services. Furthermore, increasing knowledge and education about cultural competency among all providers across the care spectrum is important.

\section{Discussion}

Despite increasing data to support evidence-based and promising rehabilitation practices, there is a dearth of research on the utilisation, outcomes and acceptability of recovery and rehabilitation services for ethnic minority patients. The limited data available suggest that rehabilitation interventions work across all patients, but outcomes may be poorer for individuals from ethnic minority groups, owing to lack of access and other barriers. Ethno-specific rehabilitation services may be highly acceptable to patients and might improve access to additional services, but there is limited evidence that they improve outcomes. Future research may benefit from utilising a community-based research methodology to work with ethnic minority patients and communities to identify problems and outcomes that are important to them. Surprisingly, there is very limited mention of racism in the recovery and rehabilitation literature, considering that it undermines communities and support, increases risk of health problems and has an impact on service provision and quality.

In some jurisdictions, data on language, ethnicity, gender and other factors that may influence access and care is not regularly collected. Such data are needed to better plan services to improve access and quality of care. There may be an argument for reorienting services and connecting patients with services where they are not currently provided or are not at an adequate level, for example in geographic areas with higher rates of SMI and greater marginalisation. There may also be a role for the reconfiguration of non-traditional rehabilitation settings, such as inpatient units and acute and emergency services, to be more rehabilitation- and recovery-oriented (Kidd 2014), particularly if they provide care to high numbers of ethnic minority patients.

Considering the poor outcomes of psychosis in both majority and minority ethnic communities it is important that all patients receive equitable social services that ensure basic income, housing and medical support. Furthermore, action must be 
taken to address inequities that exist in all social determinants of health. With the growing evidence base for rehabilitation practices, we need further research involving ethnic minority patients in highquality studies and continuing innovation in the field of rehabilitation and recovery-oriented care (which may or may not include cultural adaptation). Perhaps we will then see improved functional outcomes for all people with SMI.

\section{Conclusions}

Although there is growing evidence for rehabilitation and recovery interventions for individuals with SMI there is limited research on the utilisation, outcomes and acceptability of such services for ethnic minority patients. The limited data available suggest that rehabilitation interventions work across all patients but, because of a wide range of barriers, outcomes may be poorer for patients from ethnic minorities. Further research and focus on action to improve facilitators to rehabilitation and recovery for ethnic minority patients are needed.

\section{Acknowledgements}

The author would like to acknowledge the comments and feedback provided by Dr Kwame McKenzie and Dr Abraham Rudnick during the preparation of this manuscript.

\section{References}

Agius M, Shah S, Ramkisson R, et al (2008) Three year outcomes in an early intervention service for psychosis in a multicultural and multiethnic population. Psychiatr Danub, 20: 494-9.

Ali MK, Hack SM, Brown CH, et al (2017) Racial differences in mental health recovery among veterans with serious mental illness. J Racial Ethn Health Disparities, 5: 235-42.

Anderson KK, Cheng J, Susser E, et al (2015) Incidence of psychotic disorders among first-generation immigrants and refugees in Ontario. CMAJ, 187: E279-86.

Bernal G, Jiménez-Chafey MI, Domenech Rodríguez MM (2009) Cultural adaptation of treatments: a resource for considering culture in evidence-based practice. Prof Psychol Res Pr, 40: 361-8.

Bhugra D, Ayonrinde O, Butler G, et al (2011) A randomised controlled trial of assertive outreach $v$. treatment as usual for black people with severe mental illness. Epidemiol Psychiatr Sci, 20: 83-9

Birchwood M, Cochrane R, Macmillan F, et al (1992) The influence of ethnicity and family structure on relapse in first-episode schizophrenia: a comparison of Asian, Afro-Caribbean, and white patients. $\mathrm{Br} J$ Psychiatry, 161: 783-90.

Bond GR, Drake RE, Mueser KT, et al (2001) Assertive community treatment for people with severe mental illness. Dis Manag Health Out, 9: $141-59$

Chow W, Law S, Andermann L (2009a) ACT tailored for ethnocultural communities of metropolitan Toronto. Psychiatr Serv, 60(6): 847.

Cinnirella M, Loewenthal KM (1999) Religious and ethnic group influences on beliefs about mental illness: a qualitative interview study. $\mathrm{Br} \mathrm{J}$ Med Psychol, 72: 505-24.

Department of Health (2005) Delivering Race Equality in Mental Health Care: An Action Plan for Reform Inside and Outside Services and the
Government's Response to the Independent Inquiry into the Death of David Bennett. TSO (The Stationery Office).

Dieterich M, Irving CB, Bergman $\mathrm{H}$, et al (2017) Intensive case management for severe mental illness. Cochrane Database Syst Rev, 1: CD007906 (doi: 10.1002/14651858.CD007906.pub3).

Eack SM, Newhill CE (2012) Racial disparities in mental health outcomes after psychiatric hospital discharge among individuals with severe mental illness. Soc Work Res, 36: 41-52.

Kidd SA, Virdee G, Krupa T, et al (2013) The role of gender in housing for individuals with severe mental illness: a qualitative study of the Canadian service context. BMJ Open, 3(6): e002914.

Kidd SA, Mckenzie KJ, Virdee G (2014) Mental health reform at a systems level: widening the lens on recovery-oriented care. Can J Psychiatry, 59: 243-9.

Killaspy $\mathrm{H}$, Bebbington P, Blizard R, et al (2006) The REACT study: randomised evaluation of assertive community treatment in north London. $B M J$, 332: 815-20.

Kinoshita Y, Furukawa TA, Kinoshita K, et al (2013) Supported employment for adults with severe mental illness. Cochrane Database Syst Rev, 9: CD008297 (doi: 10.1002/14651858.CD008297.pub2).

Knifton L, Gervais M, Newbigging K, et al (2010) Community conversation: addressing mental health stigma with ethnic minority communities. Soc Psychiatry Psychiatr Epidemiol, 45: 497-504

Lukyanova VV, Balcazar EF, Oberoi AK, et al (2014) Employment outcomes among African Americans and Whites with mental illness. Work, 48: 319-28.

Morgan C, Mallett R, Hutchinson G, et al (2004) Negative pathways to psychiatric care and ethnicity: the bridge between social science and psychiatry. Soc Sci Med, 58: 739-52.

Morgan C, Fearon P, Lappin J, et al (2017) Ethnicity and long-term course and outcome of psychotic disorders in a UK sample: the EESOP-10 study. Br J Psychiatry, 211: 88-94.

Mueser KT, Bond GR, Essock SM, et al (2014) The effects of supported employment in Latino consumers with severe mental illness. Psychiatr Rehabil J, 37: 113-22.

Nagendra A, Schooler NR, Kane JM, et al (2017) Demographic, psychosocial, clinical, and neurocognitive baseline characteristics of Black Americans in the RAISE-ETP study. Schizophr Res, 193: 64-8.

Olney MF, Kennedy J (2002) Racial disparities in VR use and job placement rates for adults with disabilities. Rehabilitation Counseling Bulletin, 45: 177-85.

Revier CJ, Reininghaus U, Dutta R, et al (2015) Ten-year outcomes of firstepisode psychoses in the MRC EESOP-10 study. J Nerv Ment Dis, 203: 379-86.

Rosenheck R, Kasprow W, Frisman L, et al (2003) Cost-effectiveness of supported housing for homeless persons with mental illness. Arch Gen Psychiatry, 60: 940-51.

Rotenberg MA, Rudnick A (2011) Reporting of ethics procedures in psychiatric rehabilitation peer-reviewed empirical research publications in the last decade. Am J Psychiatr Rehabil, 14: 97-108.

Rotenberg M, Rudnick A (2017a) Recent developments in person-centered psychiatry: present and future psychiatric rehabilitation. Eur J Pers Cent Healthc, 5: 256-62.

Rotenberg M, Tuck A, McKenzie K (2017b) Psychosocial stressors contributing to emergency psychiatric service utilization in a sample of ethno-culturally diverse clients with psychosis in Toronto. BMC Psychiatry, 17(1): 324.

Sass B, Moffat J, Bhui K, et al (2009) Enhancing pathways to care for black and minority ethnic populations: a systematic review. Int Rev Psychiatry, 21: 430-8.

Shank JW, Iwasaki Y, Coyle C, et al (2015) Experiences and meanings of leisure, active living, and recovery among culturally diverse communitydwelling adults with mental illness. Am J Psychiatr Rehabil , 18: 129-51.

Stergiopoulos V, Gozdzik A, Misir V, et al (2015) Effectiveness of Housing First with intensive case management in an ethnically diverse sample of homeless adults with mental illness: a randomized controlled trial. PLoS ONE, 10(7): e0130281
MCO answers

1 a 2 b 3 c 4 c 5 e 
Tondora J, O'Connell M, Miller R, et al (2010) A clinical trial of peer-based culturally responsive person-centered care for psychosis for African Americans and Latinos. Clinical Trials, 7: 368-79.

Whitley R (2016) Ethno-racial variation in recovery from severe mental illness: a qualitative comparison. Can J Psychiatry, 61: 340-7.

Yamada A, Brekke J (2008) Addressing mental health disparities through clinical competence not just cultural competence: the need for assessment of sociocultural issues in the delivery of evidence-based psychosocial rehabilitation services. Clin Psychol Rev, 28: 1386-99.

Yang J, Law S, Chow W, et al (2005) Best practices: assertive community treatment for persons with severe and persistent mental illness in ethnic minority groups. Psychiatr Serv, 56: 1053-5.

\section{MCOs}

Select the single best option for each question stem

1 According to a Cochrane review and randomised trial, the rehabilitation practice with the strongest evidence for use with ethnic minority patients is:

a supported employment

b peer support

c cognitive remediation

d supported education

e rehabilitation assessment.

2 In conceptualising recovery, ethnic minority communities may place greater focus than White communities on:

a employment

b religion c housing

d education

e the role of pharmacotherapy.

3 Of the following, which is not a barrier to rehabilitation and recovery for ethnic minority patients?

a coercion

b stigma

c cultural adaptation of services

d location of services

e racism.

4 Ethno-specific matching has been shown to result in:

a fewer crisis presentations

b improved housing status c high family and patient satisfaction with care provided

$d$ increased length of employment tenure

e better adherence to prescribed medication.

5 Clinicians can facilitate rehabilitation with ethnic minority patients by:

a engaging spiritual and religious communities

b considering ethno-specific matching

c providing culturally responsive care

d being aware of social determinants of health

e all of the above. 